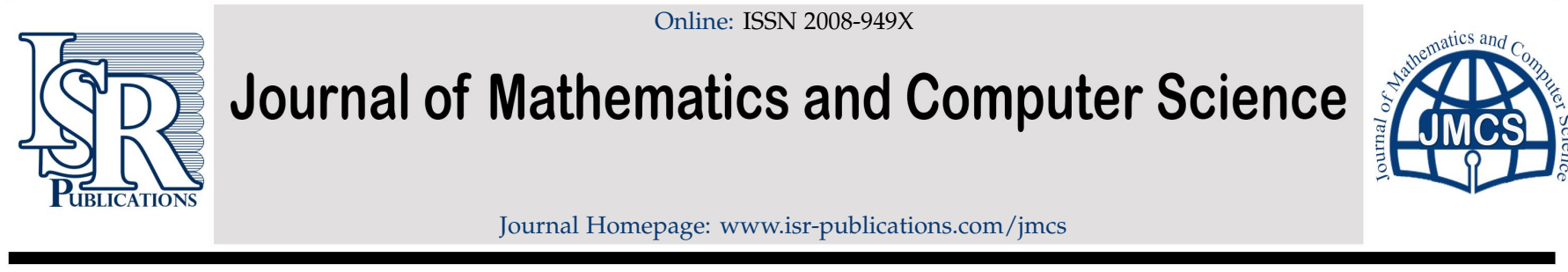

\title{
Characterizing semi-groups through the properties of their fuzzy m-bi-ideals
}

\author{
Mohammad Munira, Nasreen Kausar ${ }^{b, *}$, Salahuddin ${ }^{c}$, Anum Shafiq ${ }^{d}$, Mustafa Habib ${ }^{e}$ \\ a Department of Mathematics, Government Postgraduate College, Abbottabad, Pakistan. \\ ${ }^{b}$ Department of Mathematics and Statistics, University of Agriculture, Faisalabad, Pakistan. \\ ${ }^{c}$ Department of Mathematics, Jazan University, Jazan, Kingdom of Saudi Arabia. \\ ${ }^{d}$ School of Mathematics and Statistics, Nanjing University of Information Science and Technology, Nanjing 210044, China. \\ ${ }^{e}$ Department of Mathematics, University of Engineering and Technology, Lahore, Pakistan.
}

\begin{abstract}
In this article, we present the idea of the fuzzy m-bi-ideals in semi-groups and describe their basic algebraic properties. We also develop the forms of the fuzzy m-bi-ideals generated by an element, a subset, and a sub-semi-group of the semi-group. Important characterizations of semi-groups and their different types like m-regular semi-groups and $m$-intraregular semi-groups have been given through demonstrating examples and using properties of fuzzy m-bi-ideals in semi-groups.
\end{abstract}

Keywords: Bipotency, Fuzzy m-points, m-regular semi-groups, m-intraregular semi-groups.

2020 MSC: 20M17, 22A15.

(C)2021 All rights reserved.

\section{Introduction and preliminaries}

In this section, we first present an introduction of the research work, and then some preliminary concepts in the field of semi-group theory which are necessary to pursue the main research work in the article.

\subsection{Introduction}

semi-groups are generalizations of groups which originated in the early twentieth century. Because of their simpler structures, they comparatively possess more applications and uses in different sciences. Their uses in theoretical computer science, finite automata, graph theory, abstract evolution equations and time-invariant processes have been widespread. Ideals, being the substructures of semi-groups, are the most important components of the advanced studies in the semi-group theory. The concept of ideals was introduced by Dedekind in the theory of algebraic numbers and then generalized to one and two-sided ideals by Noether for associative rings [15]. Later mathematicians introduced the ideals in other algebraic

\footnotetext{
*Corresponding author

Email addresses: dr.mohammadmunir@gmail.com (Mohammad Munir), kausar.nasreen57@gmail.com (Nasreen Kausar)

doi: $10.22436 /$ jmcs.023.03.01
}

Received: 2020-08-26 Revised: 2020-09-08 Accepted: 2020-09-24 
structures. Originally, ideals were generalized to one-sided ideals; they were generalized to quasi ideals and quasi ideals were generalized to bi ideals. Good and Hughes introduced bi-ideals in semi-groups in 1952 [3].

Generalization of ideals in different algebraic structures $([4,5,19])$ in the context of the fuzzy set theory got impetus after the introduction of the fuzzy set by Zadeh in his landmark paper [22]. The fuzzy set theory is unique in the sense that it is able to handle numerical data as well as linguistic knowledge [15]. It models the complex systems of decision making, managerial tasks and huge networks. It is capable of handling uncertainties, non-linear and distributive processes. In 1982, Liu introduced the notion of fuzzy ideals in rings [6]. He gave more important results on the fuzzy ideals in rings in [7]. Afterwards, Mukherjee et al., in [10], Swamy et. al, in [20] and Zhang Yue in [23] gave standard results on fuzzy ideals in rings. The work on generalization of ideals were further carried out by Kumar in his article [5].

In [1], Ahsan et al., characterized semi-groups through introducing the notion of fuzzy quasi ideals. Usable results on fuzzy set theory and its applications have been also published by Zimmermann in [24], where one can find applications of fuzzy technology in information processing and dynamic data analysis. A systematic exposition of fuzzy semi-groups by Mordeson et al. is available in [9], where one can find theoretical results on fuzzy semi-groups and their uses in fuzzy coding, fuzzy finite state machines and fuzzy languages. The monograph by Mordeson and Malik [8] deals with the applications of a fuzzy approach to the concepts of automata and formal languages. Shabir et al. presented the concept of fuzzy prime bi ideals for semi-groups [17]. Xie et al., defined fuzzy ideals, left ideals, right ideals and bi-ideals generated by fuzzy sets in a semi-group [21]. Inheung Chon studied the fuzzy bi-ideal generated by a fuzzy subset in a semi-group in her article [2]. Munir et al., generalized the bi ideals for semi-groups through an index $m$ in [11]. In their paper [16], Rasuli et. al studied the fuzzy bi-ideals under $t$-norms in semi-rings. Nakkhhasen et al. introduced the notion of m-bi-hyperideals of a semihyperring as a generalization of bi-hyperideals in [14].

In this article, we define the fuzzy m-bi-ideals in semi-groups. In Subsection 1.2, we present the necessary concepts from the literature, which will be used in the course of our onward work. The idea of fuzzy m-bi-ideals and their basics properties will be discussed in the Section 2 . The forms of the finitely generated fuzzy $\mathrm{m}$ - bi ideals are presented in Section 3. Section 4 deals with the characterization of $\mathrm{m}$-regular and $\mathrm{m}$-intraregular semi-groups through their fuzzy $\mathrm{m}$-bi-ideals. The article is concluded in Section 5 .

\subsection{Preliminaries}

A set $M \neq \emptyset$ with a binary operation $*: M \times M \rightarrow M$ is known as a semi-group if it satisfies the closure and associative laws with respect to $*$. A subset $\emptyset \neq \mathrm{K} \subseteq \mathrm{M}$ is known as a sub-semi-group of $M$ if $\mathrm{K}$ itself becomes a semi-group under the operation $*$ of $M$. A sub-semi-group I of $M$ is known as a right (left) ideal if I satisfies "MI $\subseteq \mathrm{I}(\mathrm{I} M \subseteq \mathrm{I})$ ". I is an ideal of $M$ if it is both a left and a right ideal. A sub-semigroup $\mathrm{Q}$ of $\mathrm{M}$ is known as quasi ideal if it satisfies the condition $\mathrm{MQ} \cap \mathrm{QM} \subseteq \mathrm{Q}$. A sub-semi-group $\mathrm{B}$ of $M$ is termed as a bi ideal of $M$ if $B$ satisfies the condition "BMB $\subseteq$ B". For a semi-group $M$, and a positive integer $m$, we have $M^{m}=M M M \cdots M$ (m-times). $M^{s} \subseteq M^{m}$ for all positive integers $s$ and $m$, such that $s \geqslant m$. A bi ideal $B$ of a semi-group $M$ is known as m-bi-ideal of $M$ if $B$ satisfies the condition "BM ${ }^{m} B \subseteq B$ ", for a positive integer $m$, not 1 necessarily [11]. An m-quasi ideal of $M$ is a quasi-ideal of $M$ fulfilling the condition that $M^{\mathrm{m}} \mathrm{Q} \cap \mathrm{Q} \mathrm{M}^{\mathrm{m}} \subseteq \mathrm{Q}, \mathrm{m}$ is positive integer.

Definition 1.1. For a set $\emptyset \neq M$, any mapping $\lambda: M \longrightarrow[0,1]$ is known as a fuzzy set of $M$, [22].

If $\lambda$ and $\eta$ are any two fuzzy subsets of $M$, then $\lambda \leqslant \eta$ implies that $\lambda(x) \leqslant \eta(x), \forall x \in M$. $\lambda$ is a non-empty fuzzy subset if $\lambda$ is not a constant function.

For a subset, $\emptyset \neq A$, of $M$, the characteristic function, $\chi_{A}$, of $A$ is a fuzzy subset of $M$, with the values given by

$$
\chi_{A}(t)= \begin{cases}1, & \text { if } t \in A, \\ 0, & \text { if } t \notin A .\end{cases}
$$


Definition 1.2. Let $\lambda$ and $\eta$ be fuzzy subsets of semi-group $M$ [17], then

1. their disjunction, $\lambda \vee \eta$, defined by $\lambda \vee \eta(u)=\max \{\lambda(u), \eta(u)\}$;

2. their conjunction, $\lambda \wedge \eta$, defined by $\lambda \wedge \eta(u)=\min \{\lambda(u), \eta(u)\}$;

3. their composition, $\lambda \circ \eta$, given by

$$
\lambda \circ \eta(w)= \begin{cases}\sup _{w=u v}\{\min \{\lambda(u), \eta(v)\}\}, & \text { if } w \text { is expressible as } w=u v, u, v \in M, \\ 0, & \text { otherwise, }\end{cases}
$$

for all $u \in M$, are fuzzy sets of $M$.

The operation $\circ$ is given precedence over $\vee$ and $\wedge$.

Definition 1.3. A fuzzy subset $\lambda$ of a semi-group $M$ is called a fuzzy sub-semi-group of $M$ if $\lambda(u v) \geqslant$ $\min \{\lambda(u), \lambda(v)\}, \forall u, v \in M$.

Definition 1.4. With reference to [18] and [15], a fuzzy sub-semi-group $\lambda$ of a semi-group $M$ is known as a fuzzy left (right) ideal if $\chi_{M} \circ \lambda \subseteq \lambda\left(\lambda \subseteq \chi_{M} \circ \lambda\right)$. $\lambda$ is called a fuzzy bi-ideal of $M$ if it satisfies the condition " $\lambda \circ \chi_{M} \circ \lambda \subseteq \lambda$ ", or equivalently, if " $\lambda(u v w) \geqslant \min \{\lambda(u), \lambda(w)\} " \forall u, v, w \in M$. $\lambda$ is fuzzy quasi-ideal of $M$ if it satisfies the proposition " $\lambda \circ \chi_{M} \cap \chi_{M} \circ \lambda \subseteq \lambda$ ".

\section{Fuzzy m-bi-ideals}

We now develop the notion of a fuzzy m-bi-ideal in semi-groups, and present their major properties.

Definition 2.1. A fuzzy bi ideal $\lambda$ of a semi-group $M$ is known as a fuzzy m-bi-ideal of $M$ if $\lambda$ satisfies the condition " $\lambda \circ \chi_{M}{ }^{m} \circ \lambda \leqslant \lambda^{\prime}$, where

$$
\chi_{M^{m}}(t)= \begin{cases}1, & \text { if } t \in M^{m}, \\ 0, & \text { if } t \notin M^{m},\end{cases}
$$

where $m$ is a positive integer known as the bipotency $\lambda$, of $M$.

Bipotency is the value of the positive integer $m$ for which a sub-semi-group $B$ of semi-group $M$ becomes an $m$-bi-deal (see references [11-13]). In the relation $B M^{m} B \subseteq B, m$ is the bipotency. For example, 2 is the bipotency of $\mathrm{B}$ in $\mathrm{BM}^{2} \mathrm{~B} \subseteq \mathrm{B}$. It is interesting that the bipotency of an ideal of a semi-groups $M$ is one(1), whereas the bipotency of $m$-bi-ideals are 1 or greater than 1 . Our argument is based on the fact that the sub-semi-group, $M^{m}$, (sub-semi-groups of $M$ ) has more chances to satisfy the $m$-bi-ideal condition than the semi-group $M$ itself.

Note that $M^{m} \subseteq M$ and $\chi_{M^{m}}$ is a restriction of $\chi_{M}$ on $M^{m}$. An element $x \in M^{m}$ is written as $x=x_{1} x_{2} x_{3} \cdots x_{m}=\pi_{i=1}^{m} x_{i}$, where $x_{1}, x_{2}, x_{3}, \ldots, x_{m} \in M .\{0\}$ and $M$ are called the trivial fuzzy $m$-biideals; all other fuzzy $m$-bi-ideals are the non-trivial fuzzy m-bi-ideals of $M$.

Definition 2.2. A fuzzy m-quasi ideal of $M$ is the sub-semi-group of $M$ satisfying the condition " $\lambda \circ$ $\chi_{M^{m}} \wedge \chi_{M^{m}} \circ \lambda \leqslant \lambda^{\prime \prime}, m$ is a positive integer.

A fuzzy bi ideal of the semi-group $M$ is a fuzzy 1-bi ideal, whereas all the other fuzzy m-bi-ideals are to be identified by the value of $m$.

A fuzzy $m$-bi and m-quasi-ideals are primarily the sub-semi-groups of $M$. If they are just the subsets of $M$ satisfying respectively the conditions " $\lambda \circ \chi_{M^{m}} \circ \lambda \leqslant \lambda^{\prime \prime}$ and " $\lambda \circ \chi_{M^{m}} \wedge \chi_{M^{m}} \circ \lambda \leqslant \lambda^{\prime}$, they are termed as the fuzzy generalized $\mathrm{m}$-bi-ideals and fuzzy generalized $\mathrm{m}$-quasi ideals of $\mathrm{M}$. 
Example 2.3. Let $M$ be a non-empty set. The characteristic function of $M$ defined by

$$
\chi_{M}(t)= \begin{cases}1, & \text { if } t \in M, \\ 0, & \text { if } t \notin M,\end{cases}
$$

is fuzzy 1-bi ideal of M. Moreover,

$$
\chi_{M^{m}}(t)= \begin{cases}1, & \text { if } t \in M^{m}, \\ 0, & \text { if } t \notin M^{m},\end{cases}
$$

is also fuzzy $\mathrm{m}$-bi-ideal of $M$ for all $\mathrm{m}=2,3,4, \ldots$

Fuzzy $m$-quasi ideals in a semi-group are all fuzzy $m$-bi-ideals. Moreover, a fuzzy $m$-bi-ideal of $M$ is not necessarily its fuzzy bi ideals; this is evident by the following example.

Example 2.4. Consider the set $M=\left\{\left[\begin{array}{ll}a & b \\ 0 & c\end{array}\right]: a, b, c \in Q\right.$, the rational numbers $\}$, along with the usual matrix multiplication, $*$, as the binary operation. $A=\left\{\left[\begin{array}{ll}a & 0 \\ 0 & b\end{array}\right]: a, b \in Q, b \neq 0\right\}$. Then $(M, *)$ is clearly a semi-group. Define $\lambda: M \longrightarrow[0,1]$ by

$$
\lambda(t)= \begin{cases}1, & \text { if } x \in A, \\ 0, & \text { otherwise. }\end{cases}
$$

Here, $\lambda$ is not a fuzzy bi-ideals of $M$. Actually, it is fuzzy bi ideal in $M$ if $\lambda \circ \chi_{M} \circ \lambda(x) \leqslant \lambda(x) \forall x \in M$. Let $x=\left[\begin{array}{ll}2 & 3 \\ 0 & 4\end{array}\right] \in M$, and since $\left[\begin{array}{ll}2 & 3 \\ 0 & 4\end{array}\right]=\left[\begin{array}{ll}2 & 3 \\ 0 & 4\end{array}\right]\left[\begin{array}{ll}1 & 0 \\ 0 & 1\end{array}\right]$, therefore, $x \circ \lambda(x)=\sup _{x=y z}\{\min [\chi(y), \lambda(z)]\}$ for all $x \in M$, where $y=\left[\begin{array}{ll}2 & 3 \\ 0 & 4\end{array}\right]$ and $z=\left[\begin{array}{ll}1 & 0 \\ 0 & 1\end{array}\right]$ gives $\chi \circ \lambda(x)=\sup _{x=y z}\{\min [1,1]\}=1$.

Next, $\lambda \circ \chi \circ \lambda(x)=\sup _{x=w y z}\{\min [\lambda(w), x \circ \lambda(y z)]\}=1, w=\left[\begin{array}{ll}1 & 0 \\ 0 & 1\end{array}\right]$. Moreover, $\lambda(x)=0$. So, $\lambda \circ x \circ \lambda(x) \leqslant$ $\lambda(x)$ does not hold because it gives $1 \leqslant 0$. But, $\lambda$ is a fuzzy 2 -bi ideal of $M$. Here, we have

$$
M^{2}=\left\{\left[\begin{array}{cc}
a^{2}+b^{2} & b c \\
b c & c^{2}
\end{array}\right]: a, b, c \in Q\right\}
$$

Let $x \in M^{2}$, then it is clear that $\lambda \circ x_{M^{2}} \circ \lambda(x)=0$ and $\lambda(x)=0$, so $\lambda \circ x_{M^{2}} \circ \lambda(x) \leqslant \lambda(x)=0$, making $\lambda$ fuzzy 2-bi ideal of $M$.

The ideas of m-left/m-right ideals in a semi-group were presented in [11], we define their analogues in the context of fuzzy set theory.

Definition 2.5. A fuzzy subset $\lambda$ of a semi-group $M$ is called fuzzy m-left (fuzzy m-right) ideal if $\chi_{M^{m} \circ} \circ$ $\lambda \subseteq \lambda\left(\lambda \circ \chi_{M^{m}} \subseteq \lambda\right)$. A fuzzy m-ideal is both a fuzzy m-left and fuzzy m-right.

Theorem 2.6. In a semi-group $M$, any fuzzy m-left ideal or m-right ideal is also its fuzzy m-bi-ideal.

Proof. If $y \in M^{m}, y=\pi_{i=1}^{m} y_{i}$, where $y_{1}, y_{2}, y_{3}, \ldots, y_{m} \in M$. For the convenience, we take $m=2$, i.e., $y=a b$, then the result can be extended to the higher values of $m$. For a fuzzy $m$-left, $\lambda$, of $M$, then there are three cases to be discussed: 
1. When both $a$ and $b$ are in $M^{m}$, then $\chi_{M^{m}} \circ \lambda(y)=\sup _{y=a b}\left\{\min \left\{\chi_{M^{m}}(a), \lambda(b)\right\}\right\}=\sup _{y=a b}\{\min \{1, \lambda(b)\}\}=$ $\sup \{\lambda(b)\} \leqslant \sup \{\lambda(a b)\}=\lambda(y)$. i.e., $\chi_{M^{m}} \circ \lambda(y) \leqslant \lambda(y)$. Now, $\lambda \circ \chi_{M^{m}} \circ \lambda(y)=\sup \left\{\min \left\{\lambda(a), \chi_{M^{m}} \circ\right.\right.$ $y=a b \quad y=a b \quad y=a a b$

$\lambda(a b)\}\} \leqslant \sup \{\min \{\lambda(a), \lambda(a b)\}\}=\lambda(y)$. That is, $\lambda \circ \chi_{M^{m}} \circ \lambda(y) \leqslant \lambda(y)$. Similarly, this result holds if we $y=a a b$ consider $y=b a b$ or $y=a b a$ or $y=a b b$. Hence, $\lambda \circ \chi_{M^{m}} \circ \lambda(y) \leqslant \lambda(y), \forall y \in M^{m}$.

2. When ais not in $M^{m}$ and $b \in M^{m}$, then $\chi_{M^{m}} \circ \lambda(y)=\sup _{y=a b}\left\{\min \left\{\chi_{M^{m}}(a), \lambda(b)\right\}\right\}=\sup _{y=a b}\{\min \{0, \lambda(b)\}\}=$ $\sup _{y=a b}\{0\} \leqslant \sup _{y=a b}\{\lambda(a b)\}=\lambda(y)$. i.e., $\chi_{M^{m}} \circ \lambda(y) \leqslant \lambda(y)$. Now, $\lambda \circ \chi_{M^{m}} \circ \lambda(y)=\sup _{y}\left\{\min \left\{\lambda(a), \chi_{M^{m}} \circ\right.\right.$

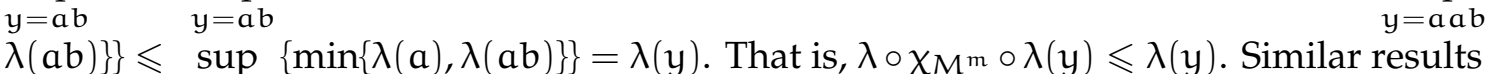
$\lambda(a b)\}\} \leqslant \sup _{y=a a b}\{\min \{\lambda(a), \lambda(a b)\}\}=\lambda(y)$. That is, $\lambda \circ \chi_{M^{m}} \circ \lambda(y) \leqslant \lambda(y)$. Similar results hold for $y=b a b$

or $y=a b a$ or $y=a b b$. Hence, $\lambda \circ \chi_{M^{m}} \circ \lambda(y) \leqslant \lambda(y), \forall y \in M^{m}$.

3. When both $a$ and $b$ are not in $M^{m}$, then $\chi_{M^{m}} \circ \lambda(y)=\sup _{y=a b}\left\{\min \left\{\chi_{M^{m}}(a), \lambda(b)\right\}\right\}=\sup _{y=a b}\{\min \{0, \lambda(b)\}\}=$ $\sup \{0\} \leqslant \sup \{\lambda(a b)\}=\lambda(y)$. i.e., $\chi_{M^{m}} \circ \lambda(y) \leqslant \lambda(y)$. Now, $\lambda \circ \chi_{M^{m}} \circ \lambda(y)=\sup \left\{\min \left\{\lambda(a), \chi_{M^{m}} \circ\right.\right.$ $y=a b$
$\lambda(a b)\}\} \leqslant \sup \{\min \{\lambda(a), \lambda(a b)\}\}=\lambda(y)$. That is, $\lambda \circ x_{M m} \circ \lambda(y) \leqslant \lambda(y)$. Similar results

$\lambda(a b)\}\} \leqslant \sup _{y=a a b}\{\min \{\lambda(a), \lambda(a b)\}\}=\lambda(y)$. That is, $\lambda \circ \chi_{M^{m}} \circ \lambda(y) \leqslant \lambda(y)$. Similar results also hold if we consider $y=b a b$ or $y=a b a$ or $y=a b b$. Hence, $\lambda \circ \chi_{M^{m}} \circ \lambda(y) \leqslant \lambda(y), \forall y \in M^{m}$.

Hence, any fuzzy $m$-left ideal of $M$ is its fuzzy $m$-bi-ideal. The case for the fuzzy m-right ideal is similar.

Corollary 2.7. A fuzzy m-ideal of $M$ is its fuzzy m-bi-ideal of $M$.

Proof. A fuzzy m-ideal of $M$, being both a fuzzy m-left and a fuzzy m-right ideal, is a fuzzy m-bi-ideal.

Theorem 2.8. Let $\lambda$ be fuzzy $m$-bi-ideal, and $\eta$ be fuzzy $n$-bi-ideal of $M$. Then their disjunction, $\lambda \wedge \eta$, defined earlier, is a fuzzy $\mathrm{p}$-bi of $\mathrm{M}$, where $\mathrm{p}=\max (\mathrm{m}, \mathrm{n})$.

Proof. Since $p=\max (m, n), M^{p} \subseteq M^{m}$ and $M^{p} \subseteq M^{n}$. We have to show $\lambda \circ \eta \circ \chi_{M^{p}}^{p} \circ \lambda \circ \eta \leqslant \lambda \circ \eta$. Take $y \in M^{p}$, then $y$ is given by $y=\pi_{i=1}^{p} y_{i}$. As discussed before, we take $p=2$, i.e., $y=a b$. We discuss three cases:

1. When both $a$ and $b$ are in $M^{p}$, then $\chi_{M^{p}} \circ \lambda \wedge \eta(y)=\sup _{y=a b}\left\{\min \left\{\chi_{M^{p}}(a), \lambda \wedge \eta(b)\right\}\right\}=\sup _{y=a b}\{\min \{1, \lambda \wedge$ $\eta(b)\}\}=\sup _{y=a b}\{\lambda \wedge \eta(b)\} \leqslant \sup _{y=a b}\{\lambda \wedge \eta(a b)\}=\lambda \wedge \eta(y)$. i.e., $x_{M^{p}} \circ \lambda \wedge \eta(y) \leqslant \lambda \wedge \eta(y)$. Now, $\lambda \wedge \eta \circ$ $\chi_{M^{p}} \circ \lambda \wedge \eta(y)=\sup _{y=a a b}\left\{\min \left\{\lambda \wedge \eta(a), \chi_{M^{p}} \circ \lambda \wedge \eta(a b)\right\}\right\} \leqslant \sup _{y=a a b}\{\min \{\lambda(\wedge \eta), \lambda \wedge \eta(a b)\}\}=\lambda \wedge \eta(y)$. That is, $\lambda \wedge \eta \circ \chi_{M^{p}} \circ \lambda \wedge \eta(y) \leqslant \lambda \wedge \eta(y)$. Similarly, this result holds if we consider $y=$ bab or $y=a b a$ or $y=a b b$. Hence, $\lambda \wedge \eta \circ \chi_{M^{p}} \circ \lambda \wedge \eta(y) \leqslant \lambda \wedge \eta(y)$ for all $y \in M^{p}$.

2. When $a \notin M^{p}$ and $b \in M^{p}$, then $\chi_{M^{p}} \circ \lambda \wedge \eta(y)=\sup _{y=a b}\left\{\min \left\{\chi_{M^{p}}(a), \lambda \wedge \eta(b)\right\}\right\}=\sup _{y=a b}\{\min \{0, \lambda \wedge$ $\eta(b)\}\}=\sup _{y=a b}\{0\} \leqslant \sup _{y=a b}\{\lambda \wedge \eta(a b)\}=\lambda \wedge \eta(y)$. i.e., $\chi_{M^{p}} \circ \lambda \wedge \eta(y) \leqslant \lambda \wedge \eta(y)$. Now, $\lambda \wedge \eta \circ \chi_{M^{p}} \circ$ $\lambda \wedge \eta(y)=\sup _{y=a a b}\left\{\min \left\{\lambda \wedge \eta(a), \chi_{M^{p}} \circ \lambda \wedge \eta(a b)\right\}\right\} \leqslant \sup _{y=a a b}\{\min \{\lambda \wedge \eta(a), \lambda \wedge \eta(a b)\}\}=\lambda \wedge \eta(y)$. That is, $\lambda \wedge \eta \circ \chi_{M^{p}} \circ \lambda \wedge \eta(y) \leqslant \lambda \wedge \eta(y)$. Similar results hold if we consider $y=b a b$ or $y=a b a$ or $y=a b b$. Hence, $\lambda \wedge \eta \circ \chi_{M^{p}} \circ \lambda \wedge \eta(y) \leqslant \lambda \wedge \eta(y)$ for all $y \in M^{p}$.

3. When both $a$ and $b$ are not in $M^{p}$, then $\chi_{M^{p}}^{p} \circ \lambda \wedge \eta(y)=\sup _{y=a b}\left\{\min \left\{\chi_{M^{p}}(a), \lambda \wedge \eta(b)\right\}\right\}=\sup _{y=a b}\{\min \{0, \lambda \wedge$ $\eta(b)\}\}=\sup _{y=a b}\{0\} \leqslant \sup _{y=a b}\{\lambda \wedge \eta(a b)\}=\lambda(y)$. i.e., $\chi_{M^{p}} \circ \lambda \wedge \eta(y) \leqslant \lambda \wedge \eta(y)$. Now, $\lambda \wedge \eta \circ \chi_{M^{p}} \circ \lambda \wedge$ $\eta(y)=\sup _{y=a a b}\left\{\min \left\{\lambda \wedge \eta(a), \chi_{M^{p}} \circ \lambda \wedge \eta(a b)\right\}\right\} \leqslant \sup _{y=a a b}\{\min \{\lambda(a) \wedge \eta, \lambda \wedge \eta(a b)\}\}=\lambda \wedge \eta(y)$. That is, 
$\lambda \wedge \eta \circ \chi_{M^{p}} \circ \lambda \wedge \eta(y) \leqslant \lambda \wedge \eta(y)$. Similar results also hold if we consider $y=b a b$ or $y=a b a$ or $y=a b b$. Hence, $\lambda \circ \chi_{M^{p}} \circ \lambda \wedge \eta(y) \leqslant \lambda \wedge \eta(y)$ for all $y \in M^{p}$.

Corollary 2.9. The intersection of fuzzy $m$-bi-ideals of a semi-group $M$ with bipotencies $m_{1}, m_{2}, \ldots$, is again a fuzzy $\mathrm{m}$-bi-ideal with bipotency $\max \left(\mathrm{m}_{1}, \mathrm{~m}_{2}, \ldots\right)$.

Corollary 2.10. If $\lambda$ is a fuzzy m-right and $\eta$ a fuzzy $n$-right ideal of a semi-group $M$, then their conjunction, $\lambda \wedge \eta$, is a fuzzy $\mathrm{p}$-bi ideal of $\mathrm{M}, \mathrm{p}=\max (\mathrm{m}, \mathrm{n})$.

Theorem 2.11. Let $\lambda$ be fuzzy $\mathrm{m}$-bi-ideal and $\eta$ be fuzzy $n$-bi-ideal of $M$. Then $\lambda \circ \eta$ is a fuzzy $p$-bi of $M$, where $p=\max (m, n)$.

Proof. Since $p=\max (m, n), M^{p} \subseteq M^{p}$ and $M^{p} \subseteq M^{n}$. We have to show $\lambda \circ \eta \circ \chi_{M^{p}}^{p} \circ \lambda \circ \eta \leqslant \lambda \circ \eta$. Take $y \in M^{p}$, then $y$ is given by $y=\pi_{i=1}^{p} y_{i}$. As before discussed before, we take $p=2$, i.e., $y=a b$. Three cases are worthy to be discussed:

1. When both $a$ and $b$ are in $M^{p}$, then $\chi_{M^{p}} \circ \lambda \circ \eta(y)=\sup _{y=a b}\left\{\min \left\{\chi_{M^{p}}(a), \lambda \circ \eta(b)\right\}\right\}=\sup _{y=a b}\{\min \{1, \lambda \circ$ $\eta(b)\}\}=\sup _{y=a b}\{\lambda \circ \eta(b)\} \leqslant \sup _{y=a b}\{\lambda \circ \eta(a b)\}=\lambda \circ \eta(y)$. i.e., $\chi_{M^{p}} \circ \lambda \circ \eta(y) \leqslant \lambda \circ \eta(y)$. Now, $\lambda \circ \eta \circ \chi_{M^{p}} \circ$ $\lambda \circ \eta(y)=\sup _{y=a a b}\left\{\min \left\{\lambda \circ \eta(a), \chi_{M^{p}} \circ \lambda \circ \eta(a b)\right\}\right\} \leqslant \sup _{y=a a b}\{\min \{\lambda(\wedge \eta), \lambda \circ \eta(a b)\}\}=\lambda \circ \eta(y)$. That is, $\lambda \circ \eta \circ \chi_{M^{p}} \circ \lambda \circ \eta(y) \leqslant \lambda \circ \eta(y)$. Similarly, this result holds if we consider $y=b a b$ or $y=a b a$ or $y=a b b$. Hence, $\lambda \circ \eta \circ \chi_{M^{p}} \circ \lambda \circ \eta(y) \leqslant \lambda \circ \eta(y), \forall y \in M^{p}$.

2. When $a \notin M^{p}$ and $b \in M^{p}$, then $\chi_{M^{p}} \circ \lambda \circ \eta(y)=\sup _{y=a b}\left\{\min \left\{\chi_{M^{p}}(a), \lambda \circ \eta(b)\right\}\right\}=\sup _{y=a b}\{\min \{0, \lambda \circ$ $\eta(b)\}\}=\sup _{y=a b}\{0\} \leqslant \sup _{y=a b}\{\lambda \circ \eta(a b)\}=\lambda \circ \eta(y)$. i.e., $\chi_{M^{p}} \circ \lambda \circ \eta(y) \leqslant \lambda \circ \eta(y)$. Now, $\lambda \circ \eta \circ \chi_{M^{p}} \circ$ $\lambda \circ \eta(y)=\sup _{y=a a b}\left\{\min \left\{\lambda \circ \eta(a), \chi_{M^{p}} \circ \lambda \circ \eta(a b)\right\}\right\} \leqslant \sup _{y=a a b}\{\min \{\lambda \circ \eta(a), \lambda \circ \eta(a b)\}\}=\lambda \circ \eta(y)$. That is, $\lambda \circ \eta \circ \chi_{M^{p}} \circ \lambda \circ \eta(y) \leqslant \lambda \circ \eta(y)$. Similar results hold if we consider $y=b a b$ or $y=a b a$ or $y=a b b$. Hence, $\lambda \circ \eta \circ \chi_{M^{p}} \circ \lambda \circ \eta(y) \leqslant \lambda \circ \eta(y), \forall y \in M^{p}$.

3. When both $a$ and $b$ are not in $M^{p}$, then $\chi_{M^{p}} \circ \lambda \circ \eta(y)=\sup _{y=a b}\left\{\min \left\{\chi_{M^{p}}(a), \lambda \circ \eta(b)\right\}\right\}=\sup _{y=a b}\{\min \{0, \lambda \circ$ $\eta(b)\}\}=\sup _{y=a b}\{0\} \leqslant \sup _{y=a b}\{\lambda \circ \eta(a b)\}=\lambda(y)$. i.e., $\chi_{M^{p}} \circ \lambda \circ \eta(y) \leqslant \lambda \circ \eta(y)$. Now, $\lambda \circ \eta \circ \chi_{M^{p}} \circ \lambda \circ$ $\eta(y)=\sup _{y=a a b}\left\{\min \left\{\lambda \circ \eta(a), \chi_{M^{p}} \circ \lambda \wedge \eta(a b)\right\}\right\} \leqslant \sup _{y=a a b}\{\min \{\lambda(a) \wedge \eta, \lambda \wedge \eta(a b)\}\}=\lambda \wedge \eta(y)$. That is, $\lambda \wedge \eta \circ \chi_{M^{p}} \circ \lambda \wedge \eta(y) \leqslant \lambda \wedge \eta(y)$. Similar results also hold if we consider $y=b a b$ or $y=a b a$ or $y=a b b$. Hence, $\lambda \circ \chi_{M^{p}} \circ \lambda \wedge \eta(y) \leqslant \lambda \wedge \eta(y), \forall y \in M^{p}$.

Lemma 2.12. If $\mathrm{A}$ and $\mathrm{B}$ are subsets of a semi-group $\mathrm{M}$, then $\chi_{\mathrm{A}} \circ \chi_{\mathrm{B}}=\chi_{\mathrm{AB}}$ (see, [17]).

Theorem 2.13. Let $A$ be a non-empty subset of semi-group $M$, such that $A \subseteq M^{m}$, then the characteristics function of $A$ is fuzzy $m$-bi ideal of $M$ if and only if $A$ is $m$-bi-ideal of $M$.

Proof. If $\chi_{A}$ is a fuzzy m-bi-ideals of $M, \chi_{A} \circ \chi_{M^{m}} \circ \chi_{A} \leqslant \chi_{A}$. By above Lemma, we have $\chi_{A M^{m}} \leqslant \leqslant \chi_{A}$. This gives that $A M^{m} A \subseteq A$, i.e., $A$ is $m$-bi ideal. The converse follows obviously.

\section{Finitely generated fuzzy m-bi-ideals in semi-groups}

The presentation of the m-bi-ideals generated by a single element, a subset and a sub-semi-group in the semi-groups have been given by Munir et al., in the article [11]. In this portion of the article, we develop the form of the fuzzy m-bi-ideals generated by a sub-semi-group, a subset and an element. 
Definition 3.1. Let $a \in M$ and $t \in(0,1]$, then the fuzzy subset of $M$, defined by

$$
a_{t}(x)= \begin{cases}t, & \text { if } x=a, \forall x \in M^{m}, \\ 0, & \text { otherwise, }\end{cases}
$$

is called a fuzzy m-point of $M$.

Definition 3.2. Let $A \subseteq M$ and $t \in(0,1]$, then the fuzzy subset of $M$, defined by

$$
A_{t}(x)= \begin{cases}t, & \text { if } x \in A, \forall x \in M^{m}, \\ 0, & \text { otherwise, }\end{cases}
$$

is called a fuzzy m-subset of $M$.

The fuzzy m-subset $A$ of $M$ is the union of the fuzzy m-points a of $M$.

Definition 3.3. Let $\lambda$ be a fuzzy subset of a semi-group $M$. Let $B_{m}(\lambda)$ denotes the intersection of all fuzzy $m$-bi-ideals of $M$ which contain $\lambda$. Then $B_{m}(\lambda)$ is a fuzzy $m$-bi-ideal of $M$, called the fuzzy m-bi-ideal generated by $\lambda$.

Theorem 3.4. Let $a_{t}$ be a fuzzy m-point of a semi-group $M$. Then the fuzzy m-bi ideal generated by $a_{t}$ denoted by $\mathrm{B}_{\mathrm{m}}\left(\mathrm{a}_{\mathrm{t}}\right)$ is defined at each $\mathrm{x} \in \mathrm{M}^{\mathrm{m}}$ by

$$
\mathrm{B}_{\mathrm{m}}\left(\mathrm{a}_{\mathrm{t}}\right)(x)= \begin{cases}\mathrm{t}, & \text { if } x \in \mathrm{B}_{\mathrm{m}}(\mathrm{a}), \\ 0, & \text { otherwise, }\end{cases}
$$

where $B_{m}(a)=\{a\} \cup\left\{a^{2}\right\} \cup\left\{a M^{m} a\right\}$, is called a fuzzy $m$-bi-ideal of $M$ generated by $a$.

Proof. Let $x \in M$, and if $y, z \in B_{m}(a)$ such that $x=y z$, then $\left(B_{m}\left(a_{t}\right)\right)^{2}(x)=V_{\{x=y z\}}\left\{B_{m}\left(a_{t}\right)(y) \wedge\right.$ $\left.B_{m}\left(a_{t}\right)(z)\right\}=t \wedge t=t=B_{m}\left(a_{t}\right)(x)$. Otherwise, $\left(B_{m}\left(a_{t}\right)\right)^{2}(x)=0 \leqslant B_{m}\left(a_{t}\right)(x)$. Thus, $\left(B_{m}\left(a_{t}\right)\right)^{2} \leqslant$ $B_{m}\left(a_{t}\right)$. Hence, $B_{m}\left(a_{t}\right)$ is a fuzzy sub-semi-group of $M$. Next, we show that $B_{m}\left(a_{t}\right)$ is a fuzzy $m$-biideal of $M$. Let $x, y, z \in M^{m}$. If $x, z \in B_{m}(a)$, then $x y z \in B_{m}(a)$ because $B_{m}(a)$ being $m$-bi-ideal. So, $B_{m}\left(a_{t}\right)(x y z)=t=B_{m}\left(a_{t}\right)(x) \wedge B_{m}\left(a_{t}\right)(z)$. If one of $x, z$ does not belong to $B_{m}(a)$, then $B_{m}\left(a_{t}\right)(x) \wedge$ $B_{m}\left(a_{t}\right)(z)=0 \leqslant B_{m}\left(a_{t}\right)(x y z)$. Thus, $B_{m}\left(a_{t}\right)(x y z) \geqslant B_{m}\left(a_{t}\right)(x) \wedge B_{m}\left(a_{t}\right)(y)$. Hence, $B_{m}\left(a_{t}\right)$ is a fuzzy $m$-bi-ideal of $M$. Also $a_{t} \leqslant B_{m}\left(a_{t}\right)$. Let $\delta$ be a fuzzy $m$-bi-ideal of $M$ containing $a_{t}$. Let $x \in M^{m}$, and if $x \notin B_{m}(a)$, then $B_{m}\left(a_{t}\right)(x)=0 \leqslant \delta(x)$. If $x \in B_{m}(a)=\{a\} \cup\left\{a^{2}\right\} \cup\left\{a M^{m} a\right\}$, then $B_{m}\left(a_{t}\right)(x)=t$. Now, if $x=a$, then $a_{t}(x)=t \leqslant \delta(x)$. If $x=a^{2}$, then if $a^{2} \in M^{m}$, then $a_{t}\left(a^{2}\right)=t$. If $a^{2} \notin M^{m}$, then $a_{t}\left(a^{2}\right)=0$. So, $a_{t}\left(a^{2}\right) \leqslant t \leqslant \delta\left(a^{2}\right)$. That is, $a_{t}\left(a^{2}\right) \leqslant \delta\left(a^{2}\right)$. If $x=$ aya for some $y \in M^{m}$, $\delta(x)=\delta($ ay $a) \geqslant \delta(a) \wedge \delta(a) \geqslant a_{t}(a) \wedge a_{t}(a)=t=B_{m}\left(a_{t}\right)(x)$. So, $\delta(x) \geqslant B_{m}\left(a_{t}\right)(x)$. Thus, $B_{m}\left(a_{t}\right)$ is the fuzzy $m$-bi-ideal containing $a_{t}$. This is the smallest such ideal of $M$.

Corollary 3.5. Let $A$ be a fuzzy subset in a semi-group $M$. Then the fuzzy $m$-bi-ideal generated by $A$ is $B_{m}(A)=$ $A \cup A^{2} \cup A M^{m} A$.

Proof. The proof is straightforward by using Definition 3.2.

Corollary 3.6. The fuzzy $m$-bi-ideal generated by a sub-semi-group $A$ of $M$ is $B_{m}(A)=A \cup A M^{m} A$.

Proof. Straight forward as $A^{2} \subseteq A$.

\section{4. m-regular and m-intra-regular semi-groups}

Let $M$ be a semi-group, an element $a \in M$ is regular if $a x a=a$ for some $x \in M$. $M$ is called a regular semi-group if every element of $M$ is regular. An element $a \in M$ is intraregular if $a=x a^{2} y$ for some $x, y \in M . M$ is called an intraregular semi-group if every element of $M$ is intraregular. 
Definition 4.1. An element a of a semi-group $M$ is known as m-regular ( $m$-intraregular) element if aya $=a$ for some $y \in M^{m}$ (if $\mathrm{ya}^{2} z=a$ for some $y, z \in M^{m}$ ). A semi-group $M$ is called m-regular ( $m$-intraregular) if every element of $M$ is $m$-regular ( $m$-intraregular).

Remark 4.2. $M$ is m-regular if $a \in a^{m} a, \forall a \in M$. It is a well-known fact that in regular semi-groups, the concepts of quasi-ideal and bi-ideal coincide. For regular semi-groups, the ideas of the fuzzy m-bi and fuzzy m-quasi-ideals coincide; proved in Theorem 4.7. Every regular (1-regular) semi-group is an m-regular semi-group, but the converse does not follow:

Example 4.3. $M=\{\Pi, \Psi, \Phi, \Xi\}$ is a semi-group with the binary operation · demonstrated in the following table.

\begin{tabular}{|c|c|c|c|c|}
\hline$\cdot$ & $\Pi$ & $\Psi$ & $\Phi$ & $\Xi$ \\
\hline$\Pi$ & $\Pi$ & $\Pi$ & $\Pi$ & $\Pi$ \\
\hline$\Psi$ & $\Pi$ & $\Psi$ & $\Phi$ & $\Psi$ \\
\hline$\Phi$ & $\Pi$ & $\Phi$ & $\Psi$ & $\Phi$ \\
\hline$\Xi$ & $\Pi$ & $\Psi$ & $\Phi$ & $\Psi$ \\
\hline
\end{tabular}

$M^{2}=\{\Pi, \Psi, \Phi\} . M$ is not regular, as, for example, $\Phi \Xi \Phi=\Phi \Phi=\Psi$, i.e., $\Xi$ is not regular. $M$ is also not intraregular, as, for example, $\Psi \Xi^{2} \Phi=\Psi \Xi \Xi \Phi=\Psi \Xi \Phi=\Psi \Phi=\Phi$, i.e., $\Xi$ is not intraregular. However, $M$ is 2-regular and 2-intraregular semi-group because $x y x=x, \forall y \in M^{2}$ and $x \in M$, and $s=t s^{2} u, \forall t, u \in M^{2}$ and $s \in M$.

Theorem 4.4. A semi-group $M$ is m-regular iff the composition and conjunction of its a fuzzy m-right and a fuzzy $\mathrm{m}$-left ideals coincide. i.e., $\lambda \circ \eta=\lambda \wedge \eta$ for a fuzzy $\mathrm{m}$-right ideal $\lambda$ and a fuzzy $\mathrm{m}$-left ideal $\eta$ of $\mathrm{M}$.

Proof. Here, $\lambda \circ \eta(x) \leqslant \lambda \circ \chi_{M^{m}}(x) \leqslant \lambda(x)$. That is, $\lambda \circ \eta(x) \leqslant \lambda(x)$ for all $x \in M^{m}$. Similarly, $\lambda \circ \eta(x) \leqslant$ $\eta(x)$. Consequently, $\lambda \circ \eta(x) \leqslant \lambda \wedge \eta(x)$. Now, if $x$ is not expressible as $x=a b$, then $\lambda \circ \eta(x)=0 \leqslant$ $\min \{\lambda(x), \eta(x)\}=\lambda \wedge \eta(x)$. So, in both the cases, $\lambda \circ \eta \leqslant \lambda \wedge \eta$.

Let $M$ is $m$-regular and $x \in M$, then $\exists y \in M^{m}$ so that $x=x y x$. When $x \in M^{m}, \lambda \circ \eta(x)=$ $\sup \min \{\lambda(x y), \eta(x)\} \geqslant \sup \min \{\lambda(x), \eta(x)\} \geqslant \min \{\lambda(x), \eta(x)\}=\lambda \wedge \eta(x)$. If $x \notin M^{m}$, then again $x=x y x$ $\lambda \circ \eta(x)=\lambda \wedge \eta(x)$. That is, $\lambda \circ \eta(x) \geqslant \lambda \wedge \eta(x)$. So, $\lambda \circ \eta \geqslant \lambda \wedge \eta$. Hence, $\lambda \circ \eta(x)=\lambda \wedge \eta(x)$. Thus, $\lambda \circ \eta=\lambda \wedge \eta$.

Conversely, let $\lambda \circ \eta=\lambda \wedge \eta$. Let $L$ be an m-left and $R$ be an m-right ideal of $M$, then $\chi_{L}$ is a fuzzy $m$ left ideal and $\chi_{R}$ a fuzzy m-right ideal of $M$. So by hypothesis, $\chi_{L} \wedge \chi_{R}=\chi_{L} \circ \chi_{R}$. This gives $\chi_{L R}=\chi_{L} \cap R$, and that $\mathrm{LR}=\mathrm{L} \cap \mathrm{R}$. So, $\mathrm{M}$ is m-regular [12].

Theorem 4.5. Let $M$ be a semi-group. Then $M$ is an $m$-regular if and only if $\lambda=\lambda \circ \chi_{M}{ }^{m} \circ \lambda$, for any fuzzy $\mathrm{m}$-bi-ideal, $\lambda$, of $\mathrm{M}$.

Proof. Suppose that $M$ to be m-regular, then for $x \in M$, there exists $y \in M^{m}$, such that $x=x y x$. There are two cases to be discussed:

1. When $x \in M^{m}$, then

$$
\begin{aligned}
\lambda \circ \chi_{M^{m}} \circ \lambda(x) & =\sup _{x=x y x}\left\{\min \left\{\lambda(x), \chi_{M^{m}} \circ \lambda(y x)\right\}\right\} \\
& =\sup _{x=x y x}\left\{\min \left\{\lambda(x), \sup _{y x=r s}\left\{\min \left\{\chi_{M^{m}}(r), \lambda(s)\right\}\right\}\right\}\right\} \\
& =\sup _{x=x y x}\left\{\min \left\{\lambda(x), \sup _{y x=r s}\{\min \{1, \lambda(s)\}\}\right\} \geqslant \sup _{x=x y x}\{\min \{\lambda(x), \lambda(x)\}\}=\lambda(x) .\right.
\end{aligned}
$$

So, $\lambda \circ \chi_{M^{m}} \circ \lambda(x) \geqslant \lambda(x)$. But, $\lambda(x) \geqslant \lambda \circ \chi_{M^{m}} \circ \lambda(x)$. Therefore, $\lambda \circ \chi_{M^{m}} \circ \lambda=\lambda$. 
2. When $x \notin M^{m}$, then $\chi_{M^{m}}(x)=0$. The rest of the result follows straightforwardly.

Conversely, if $\lambda \circ \chi_{M^{m}} \circ \lambda=\lambda$ and $a \in M$, then $\chi_{a}$ is a fuzzy m-bi-ideal of $M$, so $\chi_{a} \circ \chi_{M^{m}} \circ \chi_{a}=\chi_{a}$. This gives $a M^{m} a=a$. That is, $M$ is m-regular.

Corollary 4.6. A semi-group $M$ is $m$-regular iff $\lambda=\chi_{M^{m}} \circ \lambda \circ \chi_{M^{m}} \wedge \lambda \circ \chi_{M^{m}} \circ \lambda$, for a fuzzy $m$-bi-ideal, $\lambda$, in M.

Proof. Let $M$ be m-regular semi-group. Clearly,

$$
\chi_{M^{m}} \circ \lambda \circ \chi_{M^{m}} \wedge \lambda \circ \chi_{M^{m}} \circ \lambda \leqslant \lambda .
$$

Next,

$$
\chi_{M} \circ \lambda \circ \chi_{M}(x)=\sup _{x=x y x}\left\{\min \left\{\chi_{M^{m}}(x y), \lambda(x)\right\}\right\} \geqslant \lambda(x)
$$

and

$$
\lambda \circ \chi_{M^{m}} \circ \lambda(x) \geqslant \lambda(x)
$$

follows by Theorem 4.5. This gives,

$$
\chi_{M^{m}} \circ \lambda \circ \chi_{M^{m}} \wedge \lambda \circ \chi_{M^{m}} \circ \lambda \geqslant \lambda .
$$

Consequently,

$$
\chi_{M^{m}} \circ \lambda \circ \chi_{M^{m}} \wedge \lambda \circ \chi_{M^{m}} \circ \lambda=\lambda .
$$

The converse follows in a similar way to that of Theorem 4.5.

Theorem 4.7. In an m-regular semi-group $M$, fuzzy $m$-bi-ideal coincides with the fuzzy $m$-quasi ideal and vice versa.

Proof. If $\lambda$ be a fuzzy m-bi-ideal of m-regular semi-group $M$ and $x \in M, \lambda \circ \chi_{M^{m}} \circ \lambda(x) \leqslant \lambda(x)$. Then since $\lambda \circ \chi_{M^{m}}$ is fuzzy m-right ideal and $\chi_{M^{m}} \circ \lambda$ is fuzzy m-left ideal of $M$, so by Theorem $4.4, \lambda \circ \chi_{M^{m}} \wedge$ $\chi_{M^{m}} \circ \lambda=\lambda \circ \chi_{M^{m}} \circ \chi_{M^{m}} \circ \lambda=\lambda \circ \chi_{M^{m}} \circ \lambda \leqslant \lambda$. Thus, $\lambda \circ \chi_{M^{m}} \wedge \chi_{M^{m}} \circ \lambda \leqslant \lambda$; making $\lambda$ a fuzzy m-quasi ideal of $M$.

The following theorem is important to prove some results on fuzzy m-bi-ideals in semi-groups.

Theorem 4.8. A semi-group $M$ is $m$-regular and $m$-intraregular iff every $m$-bi-ideal, $\lambda$, is idempotent.

Proof. Straightforward.

Corollary 4.9. $M$ is $\mathrm{m}$-regular and $\mathrm{m}$-intraregular iff every $\mathrm{m}$-quasi ideal, $\eta$, is idempotent.

Proof. Straightforward.

Theorem 4.10. The following conditions are equivalent for $M$.

1. $M$ is m-intra-regular,

2. $\lambda \wedge v \leqslant \lambda \circ v$ for every fuzzy $\mathrm{m}$-right ideal, $\lambda$ and fuzzy, $\mathrm{m}$-left ideal $v$ of $M$.

Proof.

$1 \Rightarrow 2$ : Suppose $M$ is $m$-intraregular and $x \in M$, then since $M$ is $m$-intraregular, so $\exists, y, z \in M^{m}$ such that $x=y x^{2} z$. For fuzzy m-right ideal $\lambda$ and fuzzy m-left ideal $v$ of $M, \lambda \circ v(x)=\sup _{x=y x^{2} z=y x x z} \min \{\lambda(y x), v(x z)\}$ $\geqslant \min \{\lambda(x), v(x)\}=\lambda \wedge v(x)$. That is, $\lambda \circ v(x) \geqslant \lambda \wedge v(x)$. So, $\lambda \wedge v \leqslant \lambda \circ v$.

$2 \Rightarrow 1$ : Suppose $\lambda \wedge v \leqslant \lambda \circ v$. Let $L$ be an m-left and $R$ be an m-right ideal of $M$, then $\chi_{L}$ is a fuzzy $m$-left ideal and $\chi_{R}$ a fuzzy m-right ideal of $M$. So, by (2), $\chi_{L} \wedge \chi_{R} \leqslant \chi_{L} \circ \chi_{R}$. This gives $\chi_{L R} \leqslant \chi_{L} \cap R$, and that $\mathrm{LR} \leqslant \mathrm{L} \cap \mathrm{R}$. So, $M$ is m-intra-regular. 
Now, we present the following on the basis of Theorem 4.8 for the fuzzy m-bi and fuzzy m-quasiideals.

Theorem 4.11. The following conditions are equivalent for a semi-group $\mathrm{M}$ :

1. $M$ is $\mathrm{m}$-regular and $\mathrm{m}$-intra-regular;

2. $\lambda \circ \delta \geqslant \lambda \wedge \delta$, for any fuzzy m-bi-ideals $\lambda$ and $\delta$;

3. For every fuzzy $\mathrm{m}$-bi-ideal $\lambda, \lambda=\lambda \circ \lambda$;

4. For every fuzzy m-quasi-ideal $\delta, \delta=\delta \circ \delta$.

Proof.

$1 \Rightarrow 2$ : Let $a \in M$, and $M$ is m-regular and m-intra-regular, then there are $u, v, w \in M^{m}$ so that $a=a u a$ and $a=v a^{2} w$. We have $a=(a u v a)(a w u a)$, as done earlier in Theorem 4.10. Hence, $\left.\lambda \circ \delta(a)=\sup _{a=b c}\{\min \{\lambda(b), \delta(c)\}\} \geqslant \sup _{a=b c}\{\min \{\lambda(a u v a), \delta(a w u a)\}\}=\min _{a=b c}\{\lambda(b), \delta(c)\}\right\}=\lambda \wedge \delta(a)$. So, $\lambda \circ \delta(a) \geqslant$ $(\lambda \wedge \delta)(a)$. Thus, $\lambda \circ \delta \geqslant \lambda \wedge \delta$.

$2 \Rightarrow 3$ : Taking $\delta=\lambda$ in (2), we get $\lambda \circ \lambda \geqslant \lambda \wedge \lambda=\lambda$. That is $\lambda \circ \lambda \geqslant \lambda$, but $\lambda \circ \lambda \leqslant \lambda$. Consequently, $\lambda \circ \lambda=\lambda$.

$3 \Rightarrow 4$ : As every fuzzy m-quasi ideal is fuzzy $m$-bi-ideal, so by $(3), \delta \circ \delta=\delta$.

$4 \Rightarrow 1$ : Suppose $\mathrm{Q}$ is an $\mathrm{m}$-quasi-ideal of $\mathrm{M}$, then $\chi_{\mathrm{Q}}$ is a fuzzy m-quasi ideal of $M$, thus by $(3), \chi_{\mathrm{Q}} \circ \chi_{\mathrm{Q}}=$ $\chi_{\mathrm{Q}}$. This gives that $\chi_{\mathrm{QQ}}=\chi_{\mathrm{Q}}$, which implies that $\mathrm{QQ}=\mathrm{Q}$. So, by Theorem $4.10, \mathrm{M}$ is m-regular and m-intraregular.

\section{Conclusions}

In the article, the concept of the fuzzy m-bi-ideals in the semi-groups was given, along with their basic properties. It is evident that this idea and most of the results can be extended to the semi-ring theory. In a future work, we shall extend this idea to the prime, semiprime and other categories of the ideals in the semi-groups, and characterize the semi-groups through their properties. These results can be used in different fields such as computer networks, automata theory, artificial intelligence, neural net-works, signal processing, recognition processes, robotics, genetic algorithms, neural net-works, decision-making, and disease-diagnosis.

\section{References}

[1] J. Ahsan, R. M. Latif, M. Shabir, Fuzzy quasi-ideals in semi-groups, J. Fuzzy Math., 9 (2001), 259-270. 1.1

[2] I. Chon, On fuzzy bi-ideals in semi-groups, Korean J. Math., 19 (2011), 321-330. 1.1

[3] R. Good, D. Hughes, Associated groups for a semi-group, in: Bull. Amer. Math. Soc., 58 (1952), 624-625. 1.1

[4] N. Kausar, A. Meshari Alesemi, Salahuddin, M. Munir, Characterizations of non-associative ordered semi-groups by their intuitionistic fuzzy bi-ideals, Disc. Nonl. Compl., 9 (2020), 257-275. 1.1

[5] R. Kumar, Fuzzy semiprimary ideals of rings, Fuzzy Sets and Systems, 42 (1991), 263-272. 1.1

[6] W. J. Liu, Fuzzy invariant subgroups and fuzzy ideals, Fuzzy sets and Systems, 8 (1982), 133-139. 1.1

[7] W.-J. Liu, Operations on fuzzy ideals, Fuzzy Sets and Systems, 11 (1983), 31-41. 1.1

[8] J. N. Mordeson, D. S. Malik, Fuzzy automata and languages: theory and applications, Chapman \& Hall/CRC, Boca Raton, (2002). 1.1

[9] J. N. Mordeson, D. S. Malik, N. Kuroki, Fuzzy semi-groups, Springer-Verlag, Berlin, (2012). 1.1

[10] T. K. Mukherjee, M. K. Sen, On fuzzy ideals of a ring I, Fuzzy Sets and Systems, 21 (1987), 99-104. 1.1

[11] M. Munir, On m-bi ideals in semi-groups, Bull. Int. Math. Virtual Inst., 8 (2018), 461-467. 1.1, 1.2, 2, 2, 3

[12] M. Munir, A. Ali, On generalization of quasi ideals in semirings, Bull. Int. Math. Virtual Inst., 10 (2020), 83-94. 4

[13] M. Munir, A. Shafiq, A generalization of bi ideals in semirings, Bull. Int. Math. Virtual Inst., 8 (2018), 123-133. 2

[14] W. Nakkhasen, B. Pibaljommee, On m-bi-hyperideals in semihyperrings, Songklanakarin J. Sci. Technol., 41 (2019), 1241-1247. 1.1

[15] M. M. K. Rao, Fuzzy bi-interior ideals of semigroups, Asia Pacific. J. Math., 5 (2018), 208-218. 1.1, 1.4

[16] R. Rasuli, T-fuzzy bi-ideals in semirings, Earthline J. Math. Sci., 2 (2019), 241-263. 1.1 
[17] M. Shabir, Y. B. Jun, M. Bano, On prime fuzzy bi-ideals of semigroups, Iran. J. Fuzzy Syst., 7 (2010), 115-128. 1.1, 1.2, 2.12

[18] M. Shabir, Y. Nawaz, M. Aslam, Semigroups characterized by the properties of their fuzzy ideals with thresholds, World Appl. Sci. J., 14 (2011), 1851-1865. 1.4

[19] T. Shah, N. Kausar, Characterizations of non-associative ordered semigroups by their fuzzy bi-ideals, Theoret. Comput. Sci., 529 (2014), 96-110. 1.1

[20] U. M. Swamy, K. L. N. Swamy, Fuzzy prime ideals of rings, J. Math. Anal. Appl., 134 (1988), 94-103. 1.1

[21] X.-Y. Xie, Fuzzy ideals in semigroups, J. Fuzzy Math., 7 (1999), 357-365. 1.1

[22] L. A. Zadeh, Fuzzy sets, Inf. Control, 8 (1965), 338-353. 1.1, 1.1

[23] Y. Zhang, Prime L-fuzzy ideals and primary L-fuzzy ideals, Fuzzy Sets and Systems, 27 (1988), 345-350. 1.1

[24] H. Zimmermann, Fuzzy sets theory and its application, Kluwer Academic Publ., New York, (2001). 1.1 